\title{
Toxicity testing gets a makeover
}

\section{Europe aims to make chemical-exposure studies more predictive while using fewer animals.}

\section{ROME}

The European Commission has revealed details of a major new research programme to develop a modern, high-throughput approach to repeat-dose toxicity testing.

Pressure to launch such an effort arose because the commission had drafted conflicting pieces of legislation, which demanded more extensive safety testing of chemicals while also requiring less use of animals in those tests. The programme, says the commission, will help to reconcile these goals.

"Faster, cheaper and more reliable alternative methods will contribute to increased safety" while reducing the use of animals, says a commission communiqué issued in Rome last week at the World Congress on Alternatives and Animal Use in the Life Sciences, where the $€ 25$-million (US\$36-million) programme was presented.

Two items of European legislation present particular dilemmas to industry. One is the 2006 Registration, Evaluation, Authorisation and Restriction of Chemical Substances (REACH) directive, which requires retrospective testing of chemicals that are being marketed, to a point that many think overburdens existing testing capacities (see Nature $460,1065 ; 2009)$. The other is the 2003 amendment to the 1976 cosmetics directive, which phases out all testing of cosmetic ingredients on animals by 2013. The legislation also applies to imported products marketed in Europe.

Now, in the first agreement of its kind, industry will match the commission's funds through Colipa, the consortium of Europe's cosmetics, toiletry and perfumery industries based in Brussels. The total $€ 50$-million pot represents the largest-ever injection of money into the development of alternative toxicity testing.

The cosmetics industry is not particularly happy about coughing up the money when the chemicals industry is not doing the same. "Of course it is not fair," says one top representative of a cosmetics company, speaking on condition of anonymity. "But the legislation itself is not fair - the science is not there."

No one expects the new programme to be more than a modest start to the massive effort needed to rapidly and reliably test, with minimal animal use, for all possible adverse consequences of prolonged exposure to chemicals. "It will take 10 or 20 years before this is going to be translated," says meeting co-organizer Thomas Hartung, director of the Johns Hopkins University Center for Alternatives to Animal Testing in Baltimore, Maryland.

For instance, determining whether longterm exposure to a chemical causes cancer or neurological disease without using animals is much harder than the nearly completed work of replacing animals in singleexposure toxicity work. "You can't just go with a single endpoint you have to know how the whole system works," says toxicologist Horst Spielmann of the Federal Institute for Risk Assessment in Berlin.

\section{Advanced technology}

The commission's call for projects intends to incorporate expertise in five areas not widely used in traditional toxicology. These include developing methods to reliably generate other types of human cells from stem cells, and developing cellular devices that simulate organs such as the heart, lungs or kidney. Other areas include systems biology and computational modelling.

Each area will be tackled by a single consortium of researchers. "We want to concentrate the money on the minimum number of labs who can do the work needed," says Jürgen Buesing, the commission official in charge of the programme.
Stem-cell researcher Jürgen Hescheler from the University of Cologne in Germany is one of those intending to apply for funding through the initiative. "The programme puts toxicology on a new basis and brings it into the right species: the human," he says.

A US initiative - the Tox 21 programme coordinated by the Environmental Protection Agency and the National Institutes of Health - is also taking a high-throughput, systems approach to toxicology. With $\$ 22$ million for this year alone, it too aims to increase the predictive value of toxicity tests while reducing animal use, and is prioritizing chemicals most in need of testing. "It is critical that Tox 21 , and data generated in other countries, are used in Europe so that there is no duplication," says Spielmann, who is running a project under Europe's seventh framework programme for research to ensure just that.

In the meantime, scientists at the Rome meeting said that steps must be taken now to reduce the unnecessary use of animals. Bennard van Ravenzwaay, head of toxicology at the German chemicals giant BASF in Ludwigshafen, says that tests should be abandoned if they add negligible predictive value to the battery of experiments already required by regulatory agencies. Such checks include the two-generation test for reproductive toxicology, in which the second generation uses many animals without providing useful information; the mouse cancer test, which provides negligible additional information beyond the rat cancer test; and developmental neurotoxicity checks.

Regulatory authorities can also engage in "intelligent toxicity testing strategies" to reduce the number of chemicals that need full testing, says Kees van Leeuwen of TNO, the Netherlands' applied research organization in Zeist. "We can reduce which chemicals may not need a full battery of testing, by optimizing the use of information from similar chemicals," he says.

Buesing says that national agencies and industry should be prepared to extend funding of alternative methods in toxicology in the near future. "Otherwise," he says, "our $€ 50$ million will have been wasted."

Alison Abbott 